\title{
THE SUPERCONDUCTING MEDIUM BETA PROTOTYPE FOR RADIOACTIVE BEAM ACCELERATION AT TRIUMF
}

\author{
A. Facco, V. Zviagintsev, INFN-Laboratori Nazionali di Legnaro, Padova, Italy \\ R. Laxdal, TRIUMF, Vancouver, Canada \\ E. Chiaveri, CERN, Geneva, Switzerland
}

\begin{abstract}
A $106.08 \mathrm{MHz}$ superconducting quarter wave resonator, based on the bulk niobium technology developed at LNL for low beta heavy ion linacs, has been designed, constructed and tested in a collaboration between INFNLNL and TRIUMF.

This $\beta=0.072$ cavity is the medium $\beta$ prototype for the superconducting heavy ion linac of ISAC-II, the future extension to the present ISAC radioactive beam facility. The extension will also include $\beta=0.042$ and $\beta=0.105$ sections. Cavity design characteristics and test results will be reported.
\end{abstract}

\section{INTRODUCTION}

The radioactive beam facility ISAC at TRIUMF [1],[2] can produce singly charged exotic ions by means of an ISOL type source and mass-separator. A room temperature linear post-accelerator, consisting of a 35.36 MHZ RFQ and a separated function DTL working at 106.08 MHz was recently commissioned to energies variable between $0.153-1.53 \mathrm{MeV} / \mathrm{u}$ for masses $\mathrm{A} \leq 30$. TRIUMF has recently been funded to construct the ISACII upgrade to produce final energies $\mathrm{E} \geq 6.5 \mathrm{MeV} / \mathrm{u}$ for masses $\mathrm{A} \leq 150$ to allow experiments above the Coulomb barrier. ISAC-II foresees the construction of a $43 \mathrm{MV}$ superconducting booster of low, medium and high $\beta$ sections consisting of cavities with $\beta=0.042,0.072$ and 0.0105 respectively. The medium $\beta$ section will be installed first requiring twenty, two-gap cavities each producing $1 \mathrm{MV}$ acceleration assuming a design gradient of $6 \mathrm{MV} / \mathrm{m}$ at $7 \mathrm{~W}$.

The design concepts developed at LNL $[3,4]$ for the bulk niobium quarter wave resonators of ALPI and PIAVE have produced cavities with the required performance [5] over a wide range of optimum velocity. TRIUMF chose to follow a similar scheme for its superconducting booster, and a collaboration agreement between INFN and TRIUMF has been set up for the development of the ISAC-II resonator prototypes.

\section{CAVITY DESIGN AND CONSTRUCTION}

We have designed and constructed a $106.08 \mathrm{MHz}$, $\beta=0.072$ superconducting quarter wave resonator for the radioactive beam facility ISAC-II at TRIUMF. The resonator belongs to the family of the bulk niobium, low beta cavities developed for the ALPI and PIAVE linacs at
Legnaro. Their unifying characteristic is that different optimum velocities can be obtained simply by changing the resonator length, keeping all other design characteristics unchanged. Other important properties of these cavities are: the absence of an electronic fast tuner due to the presence of a mechanical damper in the inner conductor [6], the all niobium construction, and a simple machining and welding.

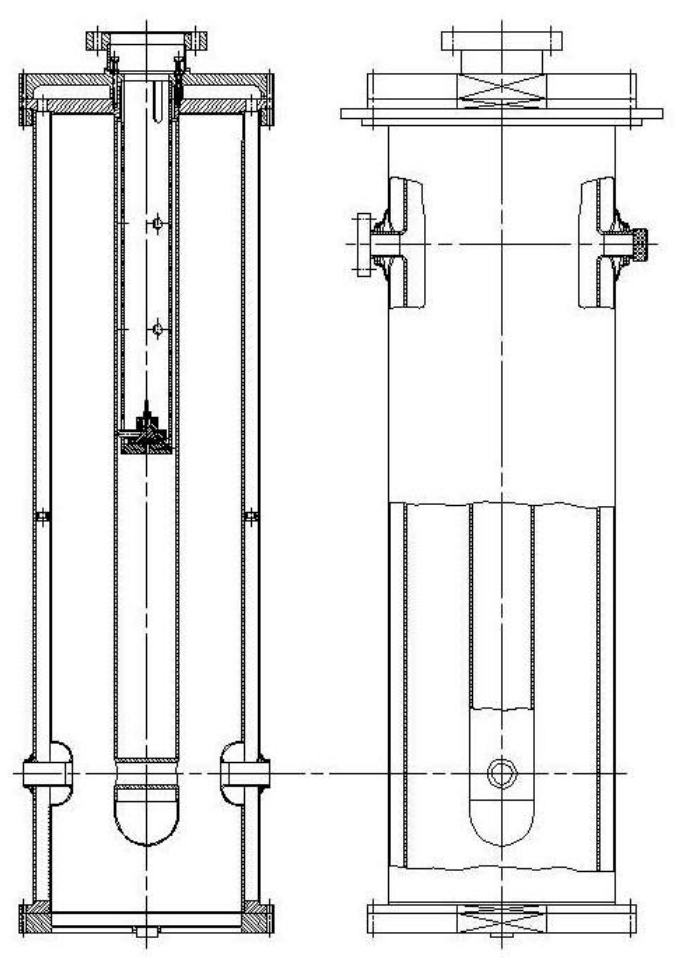

Figure1. The $106.08 \mathrm{MHz}, \beta=0.072$ cavity design

In addition to the length, matched for the required frequency of $106.08 \mathrm{MHz}$, three slight modifications have been done in comparison to the ALPI type $80 \mathrm{MHz}$ resonator design, i.e.: 1) the top flange was modified in order to increase the frequency stability against helium pressure changes; the stainless steel and the niobium plate on the top of the resonator are now more strongly connected by means of six additional screws near the inner conductor; 2) the top plate design was modified to allow a tube-to-tube, full penetration weld between the plate itself and the outer conductor; this procedure, 
although not strictly necessary, has lead to a simpler and faster operation and has reduced the risk of a welding accident; 3) instead of $\mathrm{a} \mathrm{Pb}$ or $\mathrm{Nb}$ plated copper plate machined from a thick disk, the tuning plate is made of a $1.2 \mathrm{~mm}$ niobium sheet to simplify the construction and the surface treatment.

The cavity design and the rf characteristics are shown in Fig. 1 and in Table 1, respectively.

\begin{tabular}{|c|c|c|c|}
\hline Frequency & $\mathrm{f}$ & 106.08 & $M H z$ \\
\hline Optimum velocity & $\beta_{\mathrm{o}}$ & 0.072 & \\
\hline Transit time factor at $\beta_{o}$ & $\mathrm{~T}_{\mathrm{o}}$ & 0.9 & \\
\hline Stored Energy & $\mathrm{U} / \mathrm{Ea}^{2}$ & 0.09 & $\mathrm{~J} /(\mathrm{MV} / \mathrm{m})^{2}$ \\
\hline Peak magnetic field & $\mathrm{Hp} / \mathrm{Ea}$ & $\approx 100$ & $G /(M V / m)$ \\
\hline Peak electric field & $\mathrm{Ep} / \mathrm{Ea}$ & $\approx 5$ & \\
\hline Geom. Factor $R_{s} \times Q$ & $\Gamma$ & 19.1 & $\Omega$ \\
\hline
\end{tabular}

Table 1. Cavity rf design characteristics

The mechanical construction proceeded as scheduled and the cavity was delivered by the manufacturer [7] in November 2000. During the following three months the cavity was first tested at room temperature and at $77 \mathrm{~K}$ at Legnaro, then sent to CERN for the final chemical polishing before returning to Legnaro for $\mathrm{rf}$ testing at $4.2 \mathrm{~K}$.

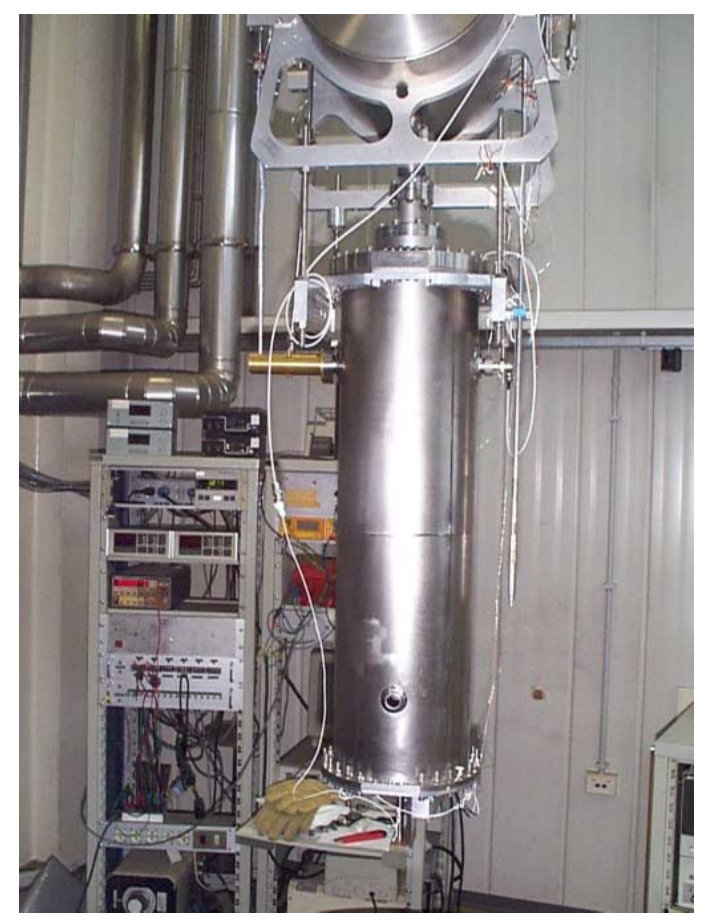

Figure 2. The $106.08 \mathrm{MHz}$ prototype before testing.

\section{TEST RESULTS}

The cavity was first tested at room temperature and at liquid nitrogen temperature, to assess mechanical and $\mathrm{rf}$ properties and to verify the good behaviour of the newly designed top flange. The frequency, according to our plans, was slightly higher than the target value, and the final adjustment was obtained by plastic deformation of the cavity in the beam port region.

The thermal contraction after cooling to $77 \mathrm{~K}$ agreed with the calculated value, and no hysteresis was observed after bringing the resonator back to room temperature. The improved connection between top niobium plate and stainless steel flange reduced by $50 \%$ the frequency pressure sensitivity, which is now about $0.9 \mathrm{~Hz} / \mathrm{mbar}$. The mechanical damper was optimized at room temperature and required a $37 \mathrm{~g}$ load, about half the weight chosen for the ALPI $80 \mathrm{MHz}$ cavities. The dangerous mechanical mode at $85 \mathrm{~Hz}$ could be damped by about $20 \mathrm{~dB}$.
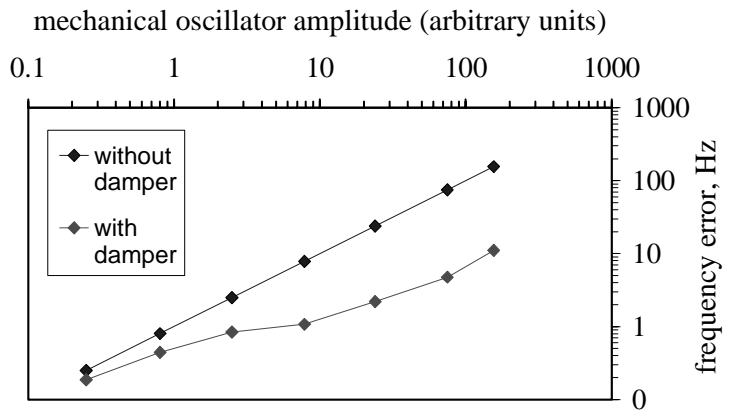

Figure 3. Results of the mechanical stability measurement at room temperature. The lowest mechanical $85 \mathrm{~Hz}$ mode was excited by a mechanical oscillator.

The first test in the superconducting regime at $4.2 \mathrm{~K}$ was done at Legnaro, after final chemical polishing performed at CERN. The multipacting conditioning started at room temperature and it was completed during resonator precooling with liquid nitrogen. Conditioning proceeded without problems and took approximately four hours. After cool down to $4.2 \mathrm{~K}$, the cavity showed significant field emission at about $6 \mathrm{MV} / \mathrm{m}$. Cavity conditioning of one hour at a pulsed rf power of $100 \mathrm{~W}$ (the maximum we could have from our amplifier) in a $3 * 10^{-5}$ mbar Helium atmosphere completely conditioned the cavity and allowed a maximum accelerating gradient of more than $11 \mathrm{MV} / \mathrm{m}$ (see Fig. 3). The $7 \mathrm{~W}$ gradient was $6.7 \mathrm{MV} / \mathrm{m}$.

No problem was observed with the thin niobium tuning plate, and the tuning range was $20 \mathrm{kHz}$.

The lower mechanical mode frequency at $4.2 \mathrm{~K}$ was about $89 \mathrm{~Hz}$, not far from the calculated value. In spite of the relatively low overcoupling allowed by our amplifier, the cavity could be locked safely to the ISAC frequency of $106.08 \mathrm{MHz}$ at the design gradient of $6 \mathrm{MV} / \mathrm{m}$. 


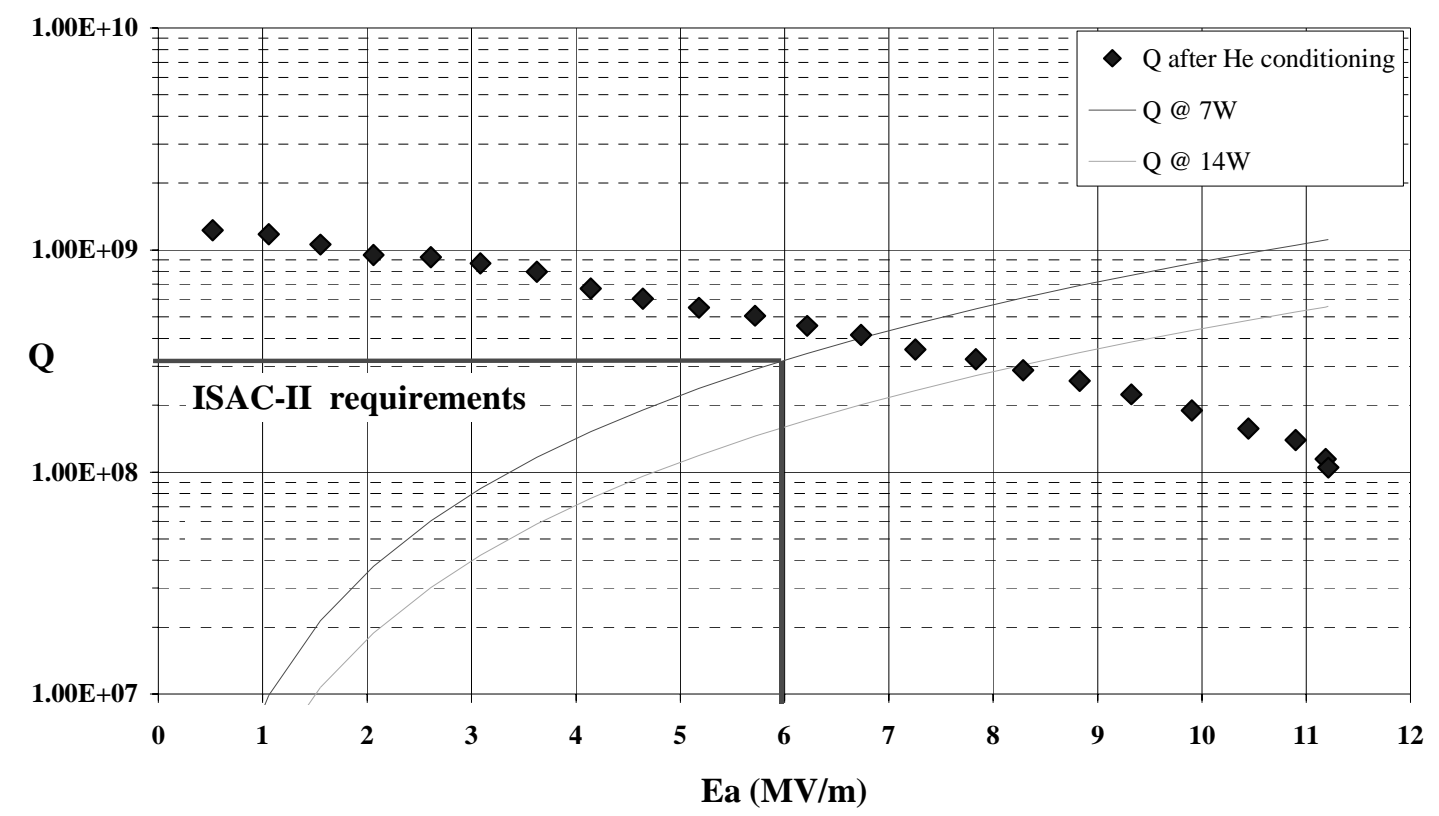

Figure 3. Performance of the ISAC-II medium beta cavity prototype at $4.2 \mathrm{~K}$.

\section{CONCLUSIONS}

The medium- $\beta$ cavity prototype of the ISAC-II superconducting linac at TRIUMF was designed, constructed and successfully tested. The cavity more than fulfilled the required specifications, exceeding the design gradient of $6 \mathrm{MV} / \mathrm{m}$ at $7 \mathrm{~W}$ and reaching more than 11 $\mathrm{MV} / \mathrm{m}$ at maximum power.

\section{ACKNOWLEDGEMENTS}

We thank S. Marigo and M. Lollo for their assistance in the preparation of the cavity tuners and in the damping load optimization, and M. Pasini for his help during the $4.2 \mathrm{~K}$ test. We are grateful also to $\mathrm{S}$. Gambalonga for his contribution in the resonator mechanical design and to $\mathrm{A}$. Insomby for the excellent chemical treatment.

\section{REFERENCES}

[1] "ISAC-II, a Project for Higher Energies at ISAC", Internal report TRI-99-1 edited by TRIUMF Publication Office, 4004 Wesbrook Mall, Vancouver, B.C. Canada V6T 2 A3.

[2] R.E. Laxdal et al., "ISAC AT TRIUMF: RECENT ACHIEVEMENTS AND FUTURE GOALS", proc. of the XX Int. Linear Accelerator Conf., Monterey, USA, 2000 .
[3] A. Facco and J.S. Sokolowski, "The Bulk Niobium Resonators Program at LNL", Proceedings of the Electrostatic accelerators and associated boosters conference, Nuclear Instruments and Methods A328(1993) 275-278.

[4] A. Facco, "Superconducting cavity development at Legnaro", Proc. of the 8th International Conference on Heavy Ion Accelerator Technology, Argonne (Chicago), USA, 1998.

[5] A. Facco, V. Zviagintsev, "Completion of the LNL Bulk Niobium Low Beta Quarter Wave Resonators", proc. of the 9th Workshop on Rf Superconductivity, Santa $\mathrm{Fe}$, USA, 1999.

[6] A. Facco, "Mechanical Mode Damping in Superconducting Low- $\beta$ Resonators", Proc. of the 8th Rf Superconductivity Workshop, Abano (Italy) 1997, Particle Accelerators, vol. 61, 265-278/1-14.

[7] http://www.zanon.com/ 